\title{
Multifocal lung cancer within ground glass lesions: Are favorable outcomes consequent to surgical resection or inherent to the disease?
}

\author{
Mara B. Antonoff, MD, FACS
}

\footnotetext{
From the Department of Thoracic and Cardiovascular Surgery, University of Texas MD Anderson Cancer Center, Houston, Tex.

Disclosures: Author has nothing to disclose with regard to commercial support.

Received for publication Aug 28, 2017; accepted for publication Aug 30, 2017; available ahead of print Oct 4 , 2017.

Address for reprints: Mara B. Antonoff, MD, FACS, 1400 Pressler St, Unit 1489, Houston, TX 77030 (E-mail: mbantonoff@mdanderson.org).

J Thorac Cardiovasc Surg 2018;155:361-2

$0022-5223 / \$ 36.00$

Copyright (C) 2017 by The American Association for Thoracic Surgery

http://dx.doi.org/10.1016/j.jtcvs.2017.08.100
}

Multiple primary lung cancer (MPLC) is a phenomenon that appears to be increasing in frequency, perhaps as a result of improved resolution of cross-sectional imaging as well as an aging population of lung cancer survivors. ${ }^{1,2}$ The diagnosis of MPLC remains complicated, however, because it must be distinguished from metastatic disease. Although criteria for identifying MPLC have been proposed and standardized, ${ }^{3,4}$ we still lack reliable and robust means of clearly discriminating patients with MPLC from those with satellite lesions and other intrapulmonary foci of metastatic disease. ${ }^{1,2}$

In their article in this issue of the Journal, Chen and colleagues $^{1}$ describe their experience with patients who underwent resection of 2 or more malignant pulmonary lesions, with widely accepted criteria used for MPLC (differing histologic type, or same histologic type with anatomic separation and $\mathrm{N}<2 \mathrm{M} 0) .{ }^{1,3,4}$ The investigators divided the patients into subgroups, with group A including patients with only ground glass opacity (GGO)-dominant lesions, group B including patients who had primary solid nodules with additional secondary GGOs, and group $\mathrm{C}$ including patients whose primary and secondary lesions were all solid in nature. After extracting samples for RNA and DNA evaluation, the investigators assessed for somatic mutations in EGFR, TP53, PIK3CA, and $B R A F$ genes, as well as EMLA-ALK, ROS1, and RET fusions. Ultimately, Chen and colleagues ${ }^{1}$ sought both to characterize differences in survival among the 3 groups and to examine extent of concordance among driver mutations between tumors within individual patients.

In this well-written article, Chen and colleagues ${ }^{1}$ describe several important findings. The 5-year recurrence-free survival was best for those with GGOs and worst for those with solid nodules $(100 \%, 68 \%$, and $51 \%$ in groups $\mathrm{A}, \mathrm{B}$, and $\mathrm{C}$, respectively). Further, such results were paralleled on analysis of the overall 5-year survival $(100 \%, 81 \%$, and $60 \%$, respectively). Chen and

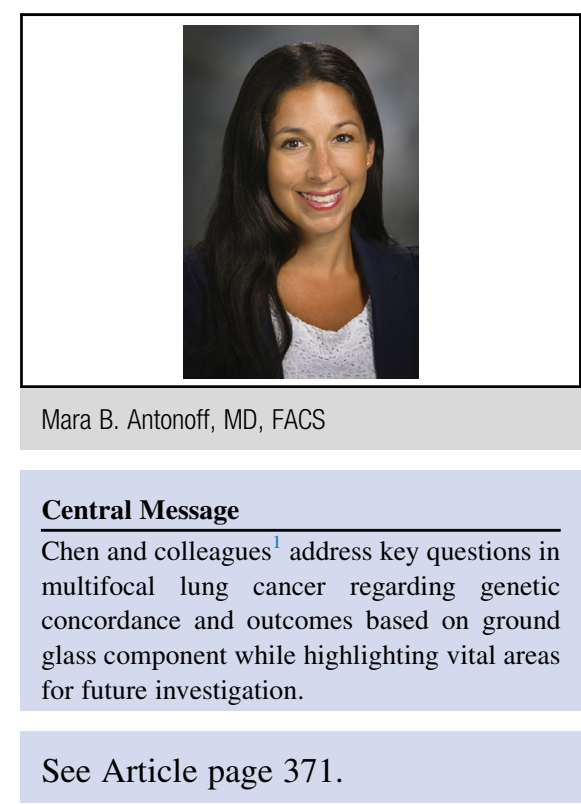

colleagues $^{1}$ clearly showed that patients with multiple GGOs had better prognoses than did those with multiple solid nodules. In a somewhat unrelated but equally important outcome of interest, Chen and colleagues ${ }^{1}$ also found a significant extent of discordance between genetic abnormalities when comparing tumors in a given individual patient ( $\sim 90 \%)$, suggesting that the secondary lesions developed as independent primary tumors rather than as intrapulmonary metastases.

In light of our current clinical environment, with increasing occurrence of MPLC cases, the findings are highly relevant to all our practices. Chen and colleagues draw on their results to generate a key take-home point. Because of their findings, Chen and colleagues ${ }^{1}$ suggest that favorable outcomes after resection support surgery as a reasonable approach to patients with MPLC, especially those with multiple GGOs. ${ }^{1}$ With regard to GGOs, recent articles have attempted to elucidate the risk of MPLC in patients with history of lung cancer and evidence of GGOs, as well as correlations with clinically aggressive behavior among patients with malignant ground glass lesions. ${ }^{5-7}$ Although such investigations are in their infancy, it has been fairly well accepted that certain patients appear to have a field effect, with multiple GGOs and a predisposition toward development of lung adenocarcinoma. Moreover, despite such tendencies in 
some individuals for neoplastic development, this has not necessarily correlated with a poorer prognosis, ${ }^{8}$ consistent with findings produced by Chen and colleagues. ${ }^{1}$

Interestingly, however, if the occurrence of multifocal disease in patients with multiple GGOs were related to a field effect, one would hypothesize that there would be greater concordance among the driver mutations of tumors within the same patient for those in group A. Nonetheless, this did not appear to be the case. Like previous investigators, Chen and colleagues ${ }^{1}$ found a high level of discrepancy among driver mutations, even within patients with multiple GGOs, similar to previous findings. ${ }^{9}$ It has been suggested that the "field effect" concept may be better explained as a consistent exposure of the field to carcinogens, rather than a specific common genetic aberration within the field. ${ }^{10}$ This argument from Gazdar and Minna ${ }^{10}$ represents just one hypothesis to explain such findings; however, like any practice-changing article, Chen's article may raise more questions than it answers.

There are a few additional interesting issues that are raised by this article. Consistent with previous authors, Chen and colleagues ${ }^{1}$ found that patients who have surgery for multifocal GGOs do quite well. ${ }^{5,9}$ This is highly important; however, one must ask whether the patients in this group are having excellent outcomes because of the surgical intervention or whether they represent a group who would fare well regardless of their treatment. We have ample literature to support the frequency of promising results for these individuals after resection but we have few data comparing surgery with other treatment modalities for this favorable group. Although we would like to consider surgical resection the reason for excellent recurrence-free and overall survivals among these patients, it may be that they are biologically destined for a better outcome regardless of therapy. It will be important to evaluate surgery versus other treatment modalities, such as targeted therapy, stereotactic radiation, and even observation, for this cohort of patients via future studies.

Another highly relevant issue raised by this article relates to the best strategy for distinguishing MPLC from metastatic disease. Chen and colleagues ${ }^{1}$ appropriately refer to previously published clinical guidelines for distinction, ${ }^{3,4}$ but they also infer that genetic evaluation such as the assays conducted in this study may more clearly separate MPLC from intrapulmonary metastases. Given that the investigators found such high genetic discordancy when clinical guidelines were used to define the cohort of interest, however, one may conclude that their detailed evaluation of genetic aberrations served to confirm the use of the existing clinical guidelines. Although it is possible that the future of our field may include genetic assays to support determinations of MPLC versus metastatic disease in our patients, it is highly reassuring that our clinical criteria may already be accurate enough to clarify such a distinction.

There are quite a few possible conclusions and take-home points from this article. It is also clear, however, that Chen and colleagues ${ }^{1}$ have provided fodder for numerous investigations to come.

\section{References}

1. Chen K, Chen W, Cai J, Yang F, Lou F, Wang X, et al. Favorable prognosis and high discrepancy of genetic features in surgical patients with multiple primary lung cancers. J Thorac Cardiovasc Surg. 2018;155:371-9.

2. Xue X, Liu Y, Pan L, Wang Y, Wang K, Zhang M, et al. Diagnosis of multiple primary lung cancer: a systematic review. J Int Med Res. 2013;41: 1779-87.

3. Martini N, Melamed MR. Multiple primary lung cancers. J Thorac Cardiovasc Surg. 1975;70:606-12.

4. Shen KR, Meyers BF, Larner JM, Jones DR, American College of Chest P. Special treatment issues in lung cancer: ACCP evidence-based clinical practice guidelines (2nd edition). Chest. 2007;132(3 Suppl):290S-305S.

5. Antonoff MB, Shewale J, Nelson D, Rice D, Sepesi B, Hofstetter W, et al. Natural history of ground glass lesions among patients with previous lung cancer: predictors of progression. Presented at: Annual Meeting of The American Association of Thoracic Surgery; April 29-May 3, 2017; Boston, MA.

6. Nelson DB, Godoy M, Benveniste M, Shewale J, Correa AM, Spicer J, et al. Clinicoradiographic predictors of aggressive behavior in resected malignant ground glass lesions. Presented at: the Annual Meeting of the Western Thoracic Surgical Association; June 21-24, 2017; Colorado Springs, CO.

7. Hattori A, Matsunaga T, Takamochi K, Oh S, Suzuki K. Prognostic impact of a ground glass opacity component in the clinical T classification of non-small cell lung cancer. J Thorac Cardiovasc Surg. 2017;154:2102-10.e1.

8. Shimada Y, Saji H, Otani K, Maehara S, Maeda J, Yoshida K, et al. Survival of a surgical series of lung cancer patients with synchronous multiple ground-glass opacities, and the management of their residual lesions. Lung Cancer. 2015; $88: 174-80$

9. Wu C, Zhao C, Yang Y, He Y, Hou L, Li X, et al. High Discrepancy of driver mutations in patients with NSCLC and synchronous multiple lung ground-glass nodules. J Thorac Oncol. 2015;10:778-83.

10. Gazdar AF, Minna JD. Multifocal lung cancers - clonality vs field cancerization and does it matter? J Natl Cancer Inst. 2009;101:541-3. 